\title{
Application of organic polymer in the identification of radioactive pu isotopes
}

\begin{abstract}
CR39 is a commercially available Poly allyl diglycol carbonate (PADC) detector which has very high efficiency in registering alpha tracks from radio nuclides. Optical properties of CR39 have been found to change in correlation with the alpha fluence incident upon it. UV-Vis spectroscopic studies have been carried out to understand these changes in alpha irradiated CR39 SSNTDs and these correlations were effectively applied for identification of radioactive $\mathrm{Pu}$ isotopes.
\end{abstract}

Volume I Issue 4 - 2017

\author{
Vrinda Devi KV,' Jayshree Ramkumar,' \\ Chandramouleeswaran $\mathrm{S}^{2}$ \\ 'Radiometallurgy Division, Bhabha Atomic Research Centre, \\ India \\ ${ }^{2}$ Analytical Chemistry Division, Bhabha Atomic Research Centre, \\ India
Correspondence: Jayshree Ramkumar, Scientific officer, Analytical Chemistry Division, Bhabha Atomic Reasearch Centre, Trombay Mumbai- 400085 India, Tel +9I-2225592224, \\ Email jrk@barc.gov.in
}

Received: August 14, 2017 | Published: September 26, 2017

\section{Introduction}

Solid state nuclear track detectors (SSNTD) are used widely in several technical applications for the detection of charged particles from protons to heavy ions, as well as the simple registration of the particle flux density or the fluencies in the environmental dosimetry. One of the most commonly used SSNTD is CR- $39^{1}$ which is a polyallyl diglycol carbonate. CR-39 is a plastic polymer commonly used in the manufacture of eyeglass lenses. The abbreviation CR 39 stands for "Columbia Resin \#39", which was the $39^{\text {th }}$ formula of a thermosetting plastic developed by the Columbia Resins project in 1940. It consists of short polyallyl chains joined with links containing carbonates and diethylene glycol groups into a dense 3D network with an initiating monomer unit. The structural formula is shown in Figure 1.

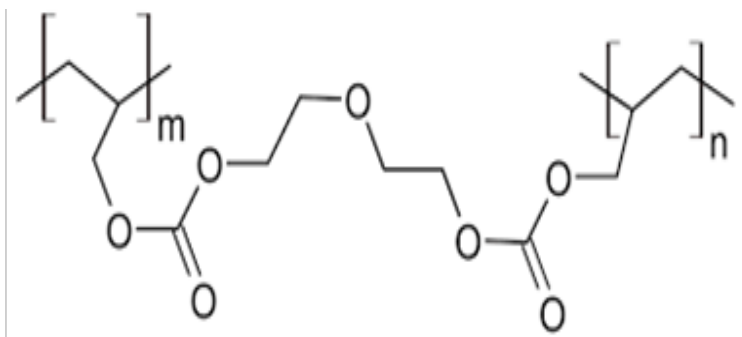

Figure I Shows the UV-visible spectra recorded for alpha irradiated CR-39 samples at different fluencies from two different sources.

Interaction of radiations results in some changes in the chemical structures and consequently in their physical and mechanical properties. Different chemical transformations like degradation, cross linking, detachment of side groups etc occur, and other chemical changes in the polymer macromolecules. Deep chemical changes occur in polymers under the action of ionizing radiations, regardless of their kind. Such radiations can, therefore, break bonds in a chain, but this does not always occur because of the redistribution and dissipation of energy. ${ }^{2,3}$ Non ionising radiations do not themselves make tracks but can have significant and sometimes profound effect on the properties of track recorders. ${ }^{4}$ When ionizing particle is incident on the film, it produces a latent track as a result of damage caused by the energy deposition of the particle. This track can be made optically visible by means of chemical etching. Two simultaneous etch rates namely the general and track, control the development of conical etch pits. The general etch rate removes the bulk of material isotropically while the track etch rate $\left(\mathrm{V}_{\mathrm{t}}\right)$ results in etch along the particle path. ${ }^{5}$ Chemical etching is the most widely used technique for revealing the latent damage trails of ionizing particles in solid. The response of a SSNTD depends strongly on the etching conditions and the etchant characteristics. The concentration of the etchant increases by using the same etchant for a longer duration due to water evaporation. ${ }^{6}$ During chemical etching, hydroxide ion hydrolyses the carbonate ester bonds and the release of polyallylalcohol from the polymer network occurs. ${ }^{7}$ In addition to the polymeric etch product polyallylalcohol (PAA), $2,2-$ Oxydiethanol is also formed in the above reaction. For polymeric detectors, the most frequently used etchant is the aqueous solution of $\mathrm{NaOH}$ with concentrations typically within the range of 1 to $12 \mathrm{~N}$ and temperature ranges from 40 to $70^{\circ} \mathrm{C} .{ }^{8}$ When $\mathrm{CR}-39$ etched in $\mathrm{NaOH}$, such polymers give rise to sodium carbonate which forms different crystalline structures depending upon its concentration in water. Other reaction products including poly-allyl alcohol, 2-2-oxy di-ethanol, allyl-alcohol and isopropyl alcohol are produced in abundance particularly during the prolonged etching of polymer detectors in $\mathrm{NaOH}$ solutions. ${ }^{9}$

Autoradiographic techniques generally are concerned with the detection of emissions from the radioactive samples. Since uranium and plutonium are alpha emitters, the alpha emission can be examined autoradiographically by using SSNTDs. The use of SSNTDs became very popular due to its advantages like insignificant changes to radiations like beta and gamma compared to alpha and also unlike the very old photographic emulsion technique it does not require darkroom for development. The tracks produced are enhanced and then captured using microscope and analyzed. Analysis of alpha images using CR39 have been earlier studied to characterise nuclear materials by correlating the registered track density with the nuclide concentrations. ${ }^{10-12}$ Alpha autoradiography technique has been primarily employed for estimating the plutonium homogeneity by detecting track clusters in the $\alpha$-image caused by the presence of 
plutonium rich agglomerates in MOX fuel. However at high alpha fluence the tracks become crowded and therefore image analysis becomes difficult. Therefore it is possible to apply to low concentration of $\mathrm{PuO}_{2}$ in fuels. Spectroscopic analysis of alpha autoradiographs was found to overcome this limitation of image analysis and hence the method applicable to characterisation of thermal as well as fast reactor fuels. ${ }^{13}$ The optical absorption method can provide information about the band structure and energy gap in crystalline and non-crystalline materials. $^{14}$

In this study, preliminary spectroscopic evaluation has been discussed which carried out to understand the change in optical properties of CR39 upon impingement by alpha from two different sources. The results of this study were perceived to be of significance for future isotopic analysis in nuclear fuels using its alpha image.

\section{Materials and methods}

Two plutonium isotopic sources namely, $\mathrm{Pu}^{239}$ and $\mathrm{Pu}^{238}$ which are both alpha emitters with $5.1 \mathrm{MeV}$ and $5.5 \mathrm{MeV}$ energy respectively were used for irradiating CR39 films of thickness $600 \mu \mathrm{m}$. The detector was topped with aluminium mylar film to cut off any non perpendicular incidence of alpha particles for better resolution. The detector was exposed to the source by placing it in close contact for the

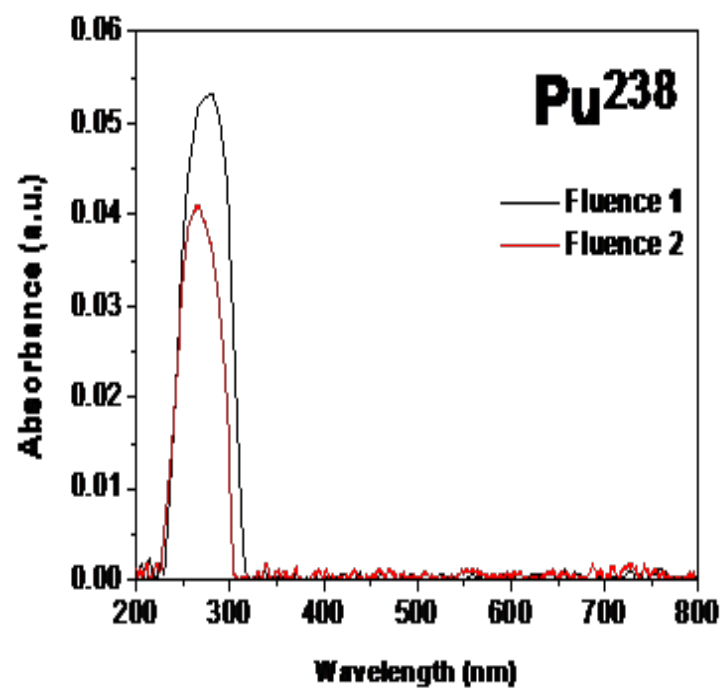

Figure 2 UVVis spectra of CR-39 irradiated with two different alpha fluences using $\mathrm{Pu}^{238}$ and $\mathrm{Pu}^{239}$ planchets

The optical band gap of the irradiated samples was determined from the spectra using Tauc equation (Equation 1) where $\alpha$ is the absorption coefficient and, hv is the energy of the incident photons, $\mathrm{E}_{\mathrm{g}}$ is the value of the optical energy gap between the valence band and the conduction band, and $r$ is an index, which characterizes the electronic transition, whether it is direct or indirect during the absorption process in the K-space.

$$
\alpha(h v)=\frac{B\left(h v-E_{g}\right)^{r}}{h v}
$$

The index $r$ can take the values 1/2,3/2, 2 and 3 for direct allowed, direct forbidden, indirect allowed and indirect forbidden transitions, respectively. The factor $\mathrm{B}$ is constant. The optical absorption coefficient, $\alpha(\mathrm{hv})$, was calculated from the absorbance, (A) as given by Equation 2.

$$
\alpha(h v)=\frac{2.303^{*} A}{d}
$$

required period of time. Chemical etching with $6 \mathrm{~N} \mathrm{KOH}$ was carried out at $72^{\circ} \mathrm{C}$ for 90 minutes to enhance the radiation induced structural modifications on CR39 film. UV-Vis spectrophotometries of etched CR39 films were carried out using JASCO V 650 spectrophotometer. The experiment was repeated for two different randomly chosen alpha fluences of $\mathrm{Pu}^{239}\left(1.86 \times 10^{5}\right.$ and $\left.2.82 \times 10^{5} \alpha / \mathrm{s}\right)$ and $\mathrm{Pu}^{238}\left(1.1 \times 10^{7}\right.$ and $\left.1.5 \times 10^{7} \alpha / \mathrm{s}\right)$ by varying the exposure time.

\section{Results and discussion}

It is seen that with increase in the alpha energy there is a shift in the peak maxima. This shift in absorbance could be attributed to the formation of possible carbon clusters or defects due to conjugation of bonds. The decrease in the peak intensity of the absorption spectra with increased alpha fluence was noticeable and this was concurrent with our earlier observations. ${ }^{13}$ It is interesting to note that the reduction in absorption of the film irradiated with $\mathrm{Pu}^{238}$ was much higher (4.5\%) as compared to that with $\mathrm{Pu}^{239}(0.5 \%)$ whereas the degree of increase in fluence values was much higher with $\mathrm{Pu}^{239}(52 \%)$ as compared to $\mathrm{Pu}^{238}$ (36\%). This could be attributed to the higher energy which is characteristic of $\mathrm{Pu}^{238}$. The decrease in intensity could be attributed to reduction in the quantum of the groups which contribute to the characteristic absorption during chemical etching assisted by alpha exposure (Figure 2).

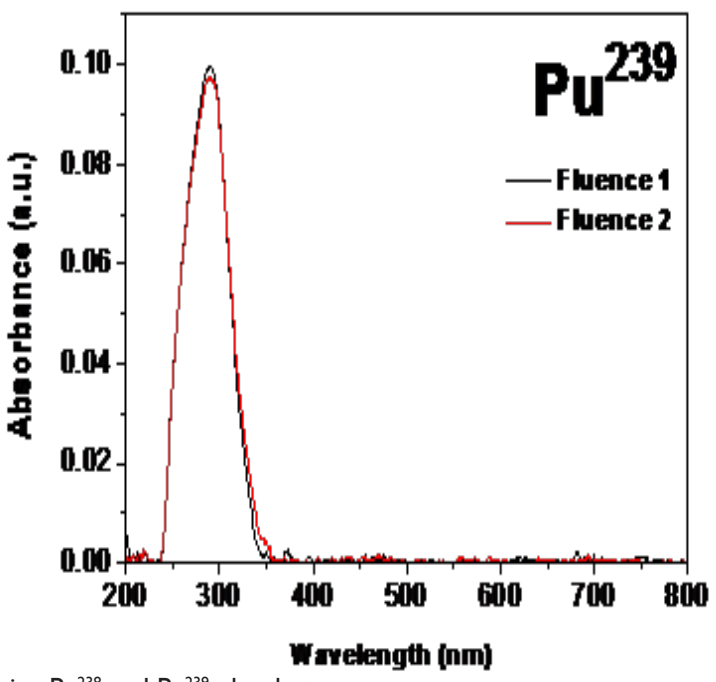

Where $\mathrm{d}$ is the sample thickness in $\mathrm{cm}$ (0.6 in this case) and A is the absorbance obtained from the peak maxima. The direct and indirect optical band gap were determined from the plots of $(\alpha h v)^{2}$ and $(\alpha h v)$ $1^{1 / 2}$ as function of photon energy hv, respectively given in Figure 3.

Extrapolating the straight parts of the relations to the hv-axis as carried out earlier ${ }^{13}$ yields the corresponding direct and indirect band gaps which are illustrated in Figure 3. The calculated band gap values for both direct and indirect transitions are given in Table 1. It is seen that a steady increase in optical band gap energy was observed with increase in $\alpha$-fluence. These studies showed that in the case of $\mathrm{Pu}^{238}$ planchet, the increase in band gap value corresponding to direct transitions is quite significant $(2.5 \%)$ in spite of smaller difference in fluence values $(36 \%)$ whereas the change is marginal for $\mathrm{Pu}^{239}(0.26 \%)$ despite higher change in fluence $(52 \%)$. 


\section{Pu-238}

Deet

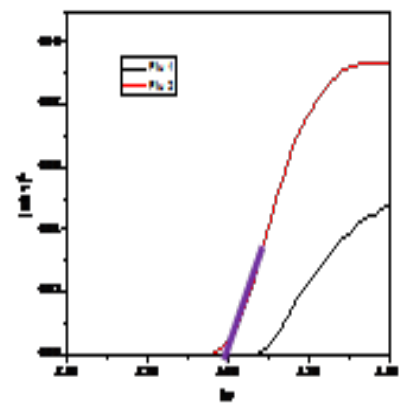

infect

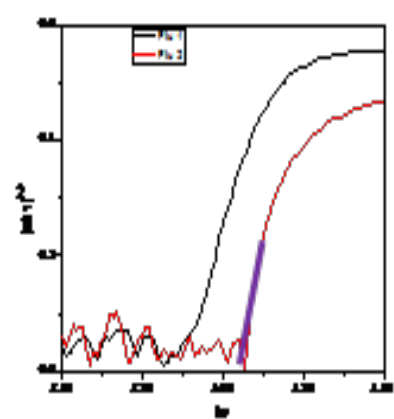

Pu-239

Diext

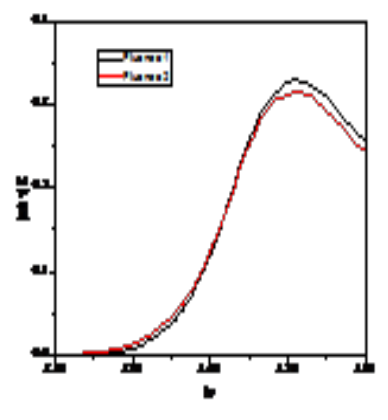

Irifd

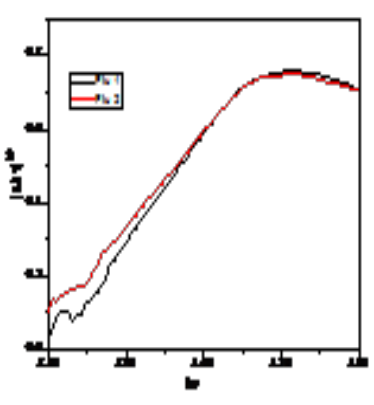

Figure 3 Band gap variations in sample irradiated with different sources.

Table I Optical band gap values for direct and indirect transitions for irradiated samples

\begin{tabular}{llll}
\hline Isotope & Fluence $(\alpha / \mathbf{s})$ & $\mathbf{E}_{\mathbf{g}}$ (Direct transition) & $\mathbf{E}_{\mathbf{g}}$ (Indirect transition) \\
\hline $\mathrm{Pu}^{238}$ & $1.1 \times 10^{7}$ & 3.95 & 3.87 \\
& $1.5 \times 10^{7}$ & 4.05 & 4.00 \\
$\mathrm{Pu}^{239}$ & $1.86 \times 10^{5}$ & $3.8 \mathrm{I}$ & 3.87 \\
& $2.82 \times 10^{5}$ & 3.82 & 4.00 \\
\hline
\end{tabular}

\section{Conclusion}

The change in optical properties of $\alpha$-irradiated CR-39 polymer could bring out useful information regarding isotopic analysis of $\mathrm{Pu}$ which is attributed to higher alpha energy. These changes are due to the cleavage of different groups within the polymer upon exposure to alpha radiations having an average energy more than $5 \mathrm{MeV}$ from different sources. A decrease in characteristic UV-Vis absorbance and increase in optical band gap were observed with increasing $\alpha$-fluence. The degree of change was dependent on the type of source used for irradiation of CR-39. This study provides further scope for the elaborate studies of changes in the properties of CR-39 to understand the different characteristics of incident alphas. This method does not generate any high-level radioactive liquid waste nor does it require any high end dedicated equipment for analysis of radionuclide.

\section{Acknowledgements}

The authors would like to express their sincere thanks to all their colleagues for their support. The authors thank Dr. PD Naik Associate Director, Chemistry Group and Shri.Vivek Bhasin, Associate Director, Nuclear Fuels Group, BARC for extending their valuable support in publishing this work.

\section{Conflict of interest}

The author declares no conflict of interest.

\section{References}

1. Ambika N, Anju S, Subhash C, et al. Modifications induced by $\mathrm{Li}^{+3}$, $\mathrm{Ni}^{+9}$ and $\mathrm{Au}^{+9}$ ion beams to $\mathrm{CR}-39$ polymer track detector. Rad Meas. 2011;46(1):127-132.

2. Abdul-Kader AM, Zaki MF, El-Badry BA. Modified the optical and electrical properties of CR-39 by gamma ray irradiation. $J$ Rad Res App Sci. 2014;7(3):286-291.
3. Cleland MR, Parks LA, Cheng S. Applications for radiation processing of materials. Nucl Instrum Methods Phys B. 2003;208:66-73.

4. Durrani SA, Bull RK. Solid state nuclear track detection principles, methods and applications. 1st ed. UK: Pergamon Press; 1987. 111:1-318.

5. Fleischer RL, Price PB, Walker RM. Nuclear tracks in solids: principles and applications. USA: University of California Press; 1975. p. 1-626.

6. Khan EU, Husaini SN, Malik F, et al. A quick method for maintaining the molarity of $\mathrm{NaOH}$ solution during continuous etching of CR-39. Rad Meas. 2002;35(1):41-45.

7. Brydson JA. Plastics based on styrene, Plastics materials. 7th ed. England: Butterworth Scientific; 1975. p. 386-422.

8. Amin SA, Henshow DL. Solid state nuclear track detectors: proceedings of the $11^{\text {th }}$ international conference. Bristol, UK; 1981. p. 7-12.

9. Husaini SN, Khan EU, Khattak NU, et al. The study of crystalline etch products of CR-39. Rad Meas. 2002;35(1):3-5.

10. Spurny F, Turek K. High-energy neutron detection with polymer nuclear track detectors. Nucl Track Detn. 1978;2(4):221-226.

11. Vilela E, Fantuzzi E, Giuacomelli G. Radiation Measurements. 1991;31:437-442.

12. Zhuo W, Tokonami S, Yonehara H, et al. A simple passive monitor for integrating measurements of indoor thoron concentrations. Rev Sci Instrum. 2002;73(8):2877-2881.

13. Vrinda Devi KV, Jayshree R, Arijit S, et al. Characterisation of nuclear fuel by spectroscopic evaluation of alpha autoradiographs. J Radioanal Nucl Chem. 2017;314(1):259-271.

14. Mishra R, Tripathy SP, Sinha D, et al. Optical and electrical properties of some electron and proton irradiated polymers. Nucl Instr Meth Phy Res B. 2000;168(1):59-64. 